\title{
Effect of fermented vegetable beverage containing Pediococcus pentosaceus in patients with mild to moderate ulcerative colitis
}

\author{
SHIGEKI BAMBA ${ }^{1}$, KENICHIRO TAKAHASHI ${ }^{2}$, HIROTSUGU IMAEDA ${ }^{2}$, ATSUSHI NISHIDA $^{2}$, \\ MASAHIRO KAWAHARA ${ }^{2}$, OSAMU INATOMI ${ }^{2}$, MITSUSHIGE SUGIMOTO ${ }^{3}$, \\ MASAYA SASAKI $^{1}$ and AKIRA ANDOH ${ }^{2}$ \\ ${ }^{1}$ Division of Clinical Nutrition, ${ }^{2}$ Department of Medicine and ${ }^{3}$ Division of Endoscopy, Shiga University of \\ Medical Science, Seta-Tsukinowa, Otsu, Shiga 520-2192, Japan
}

Received March 29, 2018; Accepted May 7, 2018

DOI: $10.3892 /$ br.2018.1099

\begin{abstract}
In patients with ulcerative colitis (UC), probiotics are often employed as an adjuvant therapeutic option. In the present study, a fermented vegetable beverage containing Pediococcus pentosaceus strain IDS885 was administered to patients with active UC for 8 weeks. A total of 11 patients with mildly to moderately active UC were randomly assigned into two groups: Group A ( $\mathrm{n}=6)$, in which the subjects consumed the fermented beverage for 8 weeks immediately following enrollment, and Group B ( $\mathrm{n}=5)$, in which the subjects were followed up for 8 weeks following enrollment and then consumed the beverage over the ensuring 8 weeks. The subjects whose Rachmilewitz clinical activity index (CAI) had decreased by $\geq 1$ point were defined as responders, whereas the subjects whose score had either been unchanged or increased were defined as non-responders. A total of 7 subjects (5 in Group A and 2 in Group B) completed the 8-week consumption regimen. No significant changes were observed in the Rachmilewitz CAI and ulcerative colitis endoscopic index of severity prior to and following the consumption period. Regarding the gastrointestinal symptom rating scale (GSRS), the total GSRS score and Question 12, 'Loose stools' were significantly improved following consumption ( $\mathrm{P}=0.042$ and 0.048 , respectively).
\end{abstract}

Correspondence to: Dr Shigeki Bamba, Division of Clinical Nutrition, Shiga University of Medical Science, Seta-Tsukinowa, Otsu, Shiga 520-2192, Japan

E-mail: sb@belle.shiga-med.ac.jp

Abbreviations: UC, ulcerative colitis; CAI, clinical activity index; GSRS, gastrointestinal symptom rating scale; T-RFLP, terminal restriction fragment length polymorphism; FMT, fecal microbiota transplantation; TNF, tumor necrosis factor; Pc. pentosaceus, Pediococcus pentosaceus; PCR, polymerase chain reaction; CFU, colony-forming units

Key words: probiotic, inflammatory bowel disease, symbiotic, short chain fatty acid, terminal restriction fragment length polymorphism
Organic acid analysis revealed that the levels of acetic acid, propionic acid and n-butyric acid tended to be continuously higher in the responders than in the non-responders. In conclusion, the fermented vegetable beverage ameliorated loose stool symptoms, although the activity of UC did not improve.

\section{Introduction}

Ulcerative colitis (UC) is a chronic inflammatory disease of unknown etiology that occurs in the large bowel (1). Previous study by our group has identified alteration of microbiota and mucosa-associated microbiota in patients with UC $(2,3)$. Probiotics are often employed as an adjuvant therapeutic strategy $(4,5)$. Clinical studies involving probiotics as the sole subject include those that have demonstrated the usefulness of Bifidobacterium-fermented milk and highly concentrated probiotic preparation (6-13). Fecal microbiota transplantation (FMT) is performed with the aim of normalizing the gut microbiota. Although single FMT has been reported to be insufficient (14), intensive FMT may be effective for patients with mild to moderately active UC (15). Since the gut microbiota is generally stable (16), bacteria that have been ingested as probiotics do not remain in the gastrointestinal tract but are excreted as transient flora (17). As such, for a probiotic therapy to be successful, it should be preferable to continuously administer a wide variety of bacterial strains in large quantities $(11,15,18)$.

In the present study, a fermented vegetable beverage containing Pediococcus pentosaceus (Pc.pentosaceus) strain IDS885 (Yasai no Senshi ${ }^{\circledR}$; Otsuka Foods Co., Ltd., Osaka, Japan) was evaluated. This beverage contains eight varieties of vegetable (equivalent to $150 \mathrm{~g}$ in weight), which are fermented with Pc.pentosaceus (a plant-derived lactic acid bacterium) to eliminate smell and improve flavor. Per serving (100 g), this fermented vegetable beverage contains $5 \times 10^{10} \mathrm{Pc}$. pentosaceus. Pc. pentosaceus is a lactic acid bacterium that can be isolated in abundance from sourdough, rainbow trout, fermented dairy products originated from India, traditional Asian fermented foods including moromi (the solid fermenting mass of swollen rice grains), mochi koji (rice cake malt) and gundruk (a fermented leafy green vegetable popular in Nepal) (19). This 
bacterium is consumed widely as part of human diet. However, to the best of our knowledge, Pc. pentosaceus has not previously been tested in patients with UC.

Herein this fermented vegetable beverage was administered to patients with active UC for 8 weeks to evaluate its effects on their gastrointestinal symptoms and environment. To assess the intestinal environment, terminal restriction fragment length polymorphism (T-RFLP) was performed for analysis of fecal microbiota and organic acids.

\section{Materials and methods}

Study design. The present study was an open-label, randomized controlled trial with approval of the Ethics Committee of Shiga University of Medical Science, Otsu, Japan (26-205, UMIN000019753). Enrolled patients had mildly to moderately active UC diagnosed according to the Mayo Score/Disease Activity Index for Ulcerative Colitis, with symptoms that had remained unchanged for at least 4 weeks between December 2015 and February 2017. Disease severity at patient enrollment was judged according to the Mayo Score/Disease Activity Index for Ulcerative Colitis, detailed previously (20). If a patient had been receiving a constant therapeutic regimen for 4 weeks prior to enrollment, concomitant use of 5-aminosalicylate preparations, prednisolone, immunomodulators, immunosuppressive agents and anti-tumor necrosis factor (TNF)- $\alpha$ antibodies was permitted throughout the trial period. Regarding anti-TNF- $\alpha$ antibodies, only patients receiving infliximab every 8 weeks or adalimumab every 2 weeks were enrolled. Patients undergoing apheresis or patients receiving antimicrobial agents were excluded. If patients experienced exacerbation of the symptoms and required additional treatment, they were withdrawn from the study. The primary endpoint was improvement of the Rachmilewitz clinical activity index (CAI), detailed previously (21), following consumption of the fermented beverage.

Consequently, 11 patients whose consent was obtained were subjected to block randomization and divided into two groups: Group A ( $n=6)$, in which the subjects consumed the fermented vegetable beverage (Yasai no Senshi ${ }^{\circledR}$; Otsuka Foods Co., Ltd.) for 8 weeks immediately following enrollment, and Group B ( $n=5)$, in which the subjects were followed up for 8 weeks following enrollment and then consumed the beverage over the ensuing 8 weeks (Fig. 1). The component composition of the fermented vegetable beverage is listed in Table I. The subjects were checked for clinical symptoms, underwent sigmoidoscopy, and fecal samples were subjected to T-RFLP and organic acid analysis immediately following enrollment, and prior to and after the full course of administration. The gastrointestinal symptom rating scale (GSRS) described previously (22) was used to evaluate the overall clinical symptoms. For the evaluation of UC activity and endoscopic severity, the Rachmilewitz CAI and uncreative colitis endoscopic index of severity were employed, respectively. For the purposes of the current study, the subjects whose Rachmilewitz CAI had decreased by $\geq 1$ point were defined as responders, whereas the subjects whose score had either been unchanged or increased were defined as non-responders.

Polymerase chain reaction (PCR) amplification and T-RFLP analysis. PCR and T-RFLP analyses were performed according to the methods described previously $(14,23)$. The $16 \mathrm{~S}$ ribosomal
Table I. Nutritional composition of fermented vegetable beverage (100 g).

\begin{tabular}{lc}
\hline Nutrient & Contents $/ 100 \mathrm{~g}$ \\
\hline Energy & $46.0 \mathrm{kcal}$ \\
Protein & $0.9 \mathrm{~g}$ \\
Lipid & $0.3 \mathrm{~g}$ \\
Carbohydrate & $10.0 \mathrm{~g}$ \\
$\mathrm{Na}^{+}$ & $23.0 \mathrm{mg}$ \\
$\mathrm{K}^{+}$ & $320.0 \mathrm{mg}$ \\
Dietary fiber & $0.8 \mathrm{~g}$ (soluble $0.4 \mathrm{~g}, \mathrm{insoluble} 0.4 \mathrm{~g}$ ) \\
Carotenoid & \\
$\alpha$-Carotene & $1,600.0 \mu \mathrm{g}$ \\
$\beta$-Carotene & $3,900.0 \mu \mathrm{g}$ \\
Lycopene & $2,000.0 \mu \mathrm{g}$ \\
Lutein & $1,130.0 \mu \mathrm{g}$ \\
Zeaxanthin & $130.0 \mu \mathrm{g}$ \\
Cryptoxanthin & $40.0 \mu \mathrm{g}$ \\
\hline
\end{tabular}

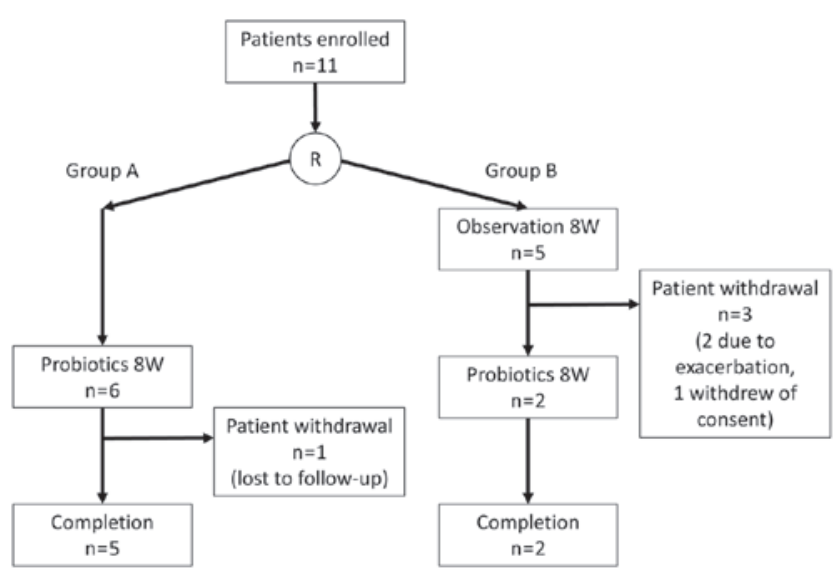

Figure 1. Flow of patients in the trial. R, randomization; W, week.

RNA gene was amplified from human fecal DNA with use of the fluorescently labeled 516F and 1510R primers (24). The PCR amplifications of DNA samples (10 ng of each DNA) were performed according to a protocol described by Nagashima et al (24).

The restriction enzyme was selected according to the Nagashima et al study (24). The purified PCR products $(2 \mu \mathrm{l})$ were digested with $20 \mathrm{U}$ of $B s l \mathrm{I}$ (Thermo Fisher Scientific, Inc., Waltham, MA, USA) at $37^{\circ} \mathrm{C}$ for $10 \mathrm{~min}$. The length of the terminal restriction fragment was determined with an ABI PRISM 3130xl genetic analyzer (Applied Biosystems; Thermo Fisher Scientific, Inc.) in GeneMapper mode. The fragment size was estimated with use of the local Southern method taxonomic units as described by Nagashima et al (24). The major terminal restriction fragments were identified by Human Fecal Microbiota T-RFLP profiling (https://www. tecsrg.co.jp/t-rflp/t_rflp_hito_OTU.html).

Measurement of $p H$ and organic acids. Following sampling, the fecal $\mathrm{pH}$ of fecal samples was immediately measured 
Table II. Clinical characteristics of patients $(n=11)$.

\begin{tabular}{|c|c|c|c|}
\hline Characteristic & Group A & Group B & P-value \\
\hline Male:female & $3: 3$ & $2: 3$ & $1.000^{\mathrm{a}}$ \\
\hline Age, median (IQR) & $43(36-48)$ & $58(39-60)$ & $0.429^{\mathrm{b}}$ \\
\hline Disease duration, years, median (IQR) & $11.6(4.7-12.1)$ & $10.0(2.5-20.5)$ & $0.792^{\mathrm{b}}$ \\
\hline Behavior (first attack/relapse-remitting/chronic-persistent) & $1 / 3 / 2$ & $0 / 3 / 2$ & $0.632^{\mathrm{a}}$ \\
\hline Location (pancolitis/left-sided/proctitis) & $3 / 1 / 2$ & $3 / 2 / 0$ & $0.323^{\mathrm{a}}$ \\
\hline Rachmilewitz CAI, median (IQR) & $4(3.0-4.8)$ & $3(2.0-4.0)$ & $0.256^{\mathrm{b}}$ \\
\hline Mayo score at baseline, median (IQR) & $6(4.3-6.0)$ & $5(4.0-5.0)$ & $0.562^{\mathrm{b}}$ \\
\hline UCEIS, median (IQR) & $4(3.0-4.8)$ & $3(3.0-4.0)$ & $0.762^{\mathrm{b}}$ \\
\hline \multicolumn{4}{|l|}{ Medication } \\
\hline 5-ASA (time-dependent/pH-dependent) & $6 / 0$ & $3 / 2$ & $0.353^{\mathrm{a}}$ \\
\hline Azathioprine (yes/no) & $2 / 4$ & $2 / 3$ & $1.000^{\mathrm{a}}$ \\
\hline Probiotics (yes/no) & $2 / 4$ & $2 / 3$ & $1.000^{\mathrm{a}}$ \\
\hline
\end{tabular}

${ }^{\mathrm{a}} \chi^{2}$ test. ${ }^{b}$ Mann-Whitney's U test. IQR, interquartile range; CAI, clinical activity index; UCEIS, ulcerative colitis endoscopic index of severity; 5-ASA, 5-amynosalicylate.

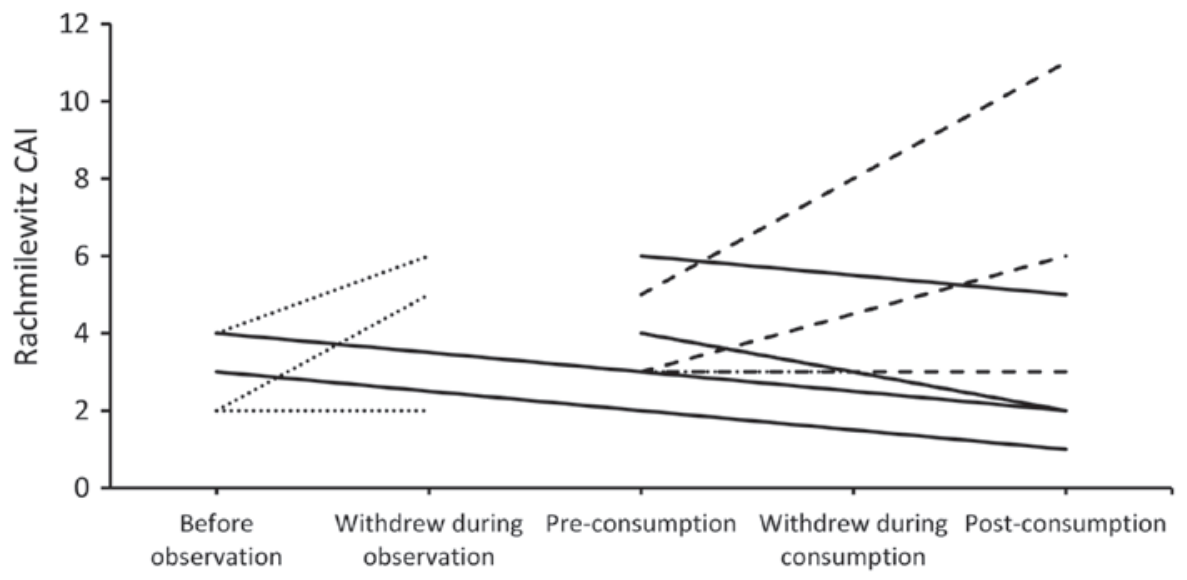

Figure 2. Disease activity of all patients in the trial. The solid lines indicate patients in which Rachmilewitz CAI declined following consumption (responders); the broken lines indicate patients in which Rachmilewitz CAI remained unchanged or increased (non-responders); the dotted lines indicate dropout cases. CAI, clinical activity index.

with a handy digital $\mathrm{pH}$ meter (KS701; Shindengen Electric Manufacturing Co., Ltd., Tokyo, Japan). Fecal samples were sterilized at $80^{\circ} \mathrm{C}$ for 15 min with Milli-Q water (Merck KGaA, Darmstadt, Germany) and then homogenized. Following centrifugation at $15,350 \mathrm{x}$ g for $10 \mathrm{~min}$ at room temperature, the supernatant fluid was passed through a $0.45-\mu$ m membrane filter and assigned for measurement of 9 short chain fatty acids (succinic, lactic, formic, acetic, propionic, iso-butyric, n-butyric, iso-valeric and n-valeric) by high performance liquid chromatography at Techno Suruga Laboratory Co., Ltd. (Shizuoka, Japan).

Statistical analysis. The data were presented as median (interquartile range). All statistical analyses were performed using Prism version 6.05 (GraphPad Software Inc., La Jolla, CA, USA). The $\chi^{2}$ test and Mann-Whitney's U test were used to compare the clinical characteristics of patients between groups A and B. The Wilcoxon signed-rank test was used to compare changes in measured parameters prior to and following consumption of the fermented vegetable beverage. $\mathrm{P}<0.05$ was regarded to indicate statistical significance.

\section{Results}

Study participants. The clinical characteristics of the 11 patients who consented to participate in the study are listed in Table II. Group A patients $(n=6)$ were required to start consuming the fermented vegetable beverage immediately following enrollment, and Group B patients $(n=5)$ started consuming the beverage when the follow-up period ended. In Group A, 1 subject became unfollowable midway through due to geographic relocation; however, exhibited unchanged clinical symptoms at their final examination at 4 weeks after the start of consumption. In group B, 2 of the 5 subjects required additional treatment during the follow-up period and had to be withdrawn. Another subject canceled their participation 
Table III. Changes in clinical parameters.

\begin{tabular}{|c|c|c|c|}
\hline & Pre-consumption & Post-consumption & P-value \\
\hline Rachmilewitz CAI & $3(3.0-4.5)$ & $3(2.0-5.5)$ & 1.000 \\
\hline UCEIS & $3(3.0-4.0)$ & $4(2.5-4.0)$ & 1.000 \\
\hline Mayo score & $5(4.0-6.0)$ & $5(3.5-5.5)$ & 1.000 \\
\hline Bowel movement & $1(0.5-2.0)$ & $1(0.5-1.5)$ & 1.000 \\
\hline Rectal bleeding & $1(0.0-1.0)$ & $1(0.0-1.0)$ & 0.773 \\
\hline Endoscopic score & $2(2.0-2.0)$ & $2(2.0-2.0)$ & 1.000 \\
\hline General appearance & $1(1.0-1.0)$ & $1(1.0-1.0)$ & 1.000 \\
\hline GSRS total score & $35(29.8-40.3)$ & $24(24.0-31.0)$ & 0.042 \\
\hline $\begin{array}{l}\text { Pain or discomfort in your upper abdomen } \\
\text { or pit of the stomach }\end{array}$ & $1(1.0-2.0)$ & $1(1.0-1.0)$ & 0.710 \\
\hline Heartburn & $1(1.0-1.5)$ & $1(1.0-2.0)$ & 1.000 \\
\hline Acid reflux & $1(1.0-1.5)$ & $1(1.0-2.0)$ & 1.000 \\
\hline Hunger pains & $1(1.0-2.0)$ & $1(1.0-2.0)$ & 1.000 \\
\hline Nausea & $1(1.0-2.0)$ & $1(1.0-1.0)$ & 0.174 \\
\hline Rumbling & $1(1.0-2.0)$ & $1(1.0-1.5)$ & 1.000 \\
\hline Bloated & $1(1.0-3.0)$ & $1(1.0-1.0)$ & 1.000 \\
\hline Burping & $1(1.0-2.0)$ & $1(1.0-1.0)$ & 1.000 \\
\hline Passing gas or flatus & $3(1.5-4.0)$ & $2(2.0-3.0)$ & 0.577 \\
\hline Constipation & $1(1.0-3.0)$ & $1(1.0-1.0)$ & 0.371 \\
\hline Diarrhea & $4(3.5-5.0)$ & $3(1.0-3.0)$ & 0.057 \\
\hline Loose stools & $4(2.5-4.0)$ & $3(1.5-3.0)$ & 0.048 \\
\hline Hard stools & $1(1.0-2.5)$ & $1(1.0-1.0)$ & 0.423 \\
\hline Urgent need to have a bowel movement & $4(3.5-6.0)$ & $2(1.5-3.5)$ & 0.100 \\
\hline Sensation of not completely emptying the bowels & $3(2.0-4.5)$ & $2(1.0-2.5)$ & 0.269 \\
\hline Fecal pH & $6.25(5.81-7.21)$ & $6.45(6.10-6.67)$ & 1.000 \\
\hline \multicolumn{4}{|l|}{$\begin{array}{l}\text { Fecal organic acids, \% positive detection } \\
\text { and median } \mathrm{mg} / \mathrm{g}(\mathrm{IQR})\end{array}$} \\
\hline Succinic acid & $57 \%, 0.20(0.15-0.55)$ & $57 \%, 0.12(0.11-0.51)$ & 0.438 \\
\hline Lactic acid & $57 \%, 0.88(0.30-2.20)$ & $43 \%, 0.55(0.36-0.73)$ & 0.375 \\
\hline Acetic acid & $100 \%, 1.76(1.48-2.76)$ & $100 \%, 1.64(0.92-3.06)$ & 0.813 \\
\hline Propionic acid & $100 \%, 0.52(0.40-0.99)$ & $100 \%, 0.41(0.31-1.33)$ & 0.813 \\
\hline iso-butyric acid & $0 \%$ & $14 \%, 0.12(0.12-0.12)$ & 1.000 \\
\hline n-butyric acid & $100 \%, 0.34(0.19-0.39)$ & $100 \%, 0.48(0.21-0.65)$ & 0.578 \\
\hline iso-valeric acid & $14 \%, 0.10(0.10-0.10)$ & $43 \%, 0.19(0.15-0.19)$ & 0.265 \\
\hline \multicolumn{4}{|l|}{ T-RFLP, occupancy, \% } \\
\hline Bifidobacterium & $26.28(10.56-31.48)$ & $16.11(12.35-27.57)$ & 0.109 \\
\hline Lactobacillales & $8.85(6.86-18.20)$ & $5.19(4.28-15.14)$ & 0.578 \\
\hline Bacteroides & $29.39(14.53-40.37)$ & $31.97(23.00-41.80)$ & 1.000 \\
\hline Prevotella & $0.00(0.00-0.00)$ & $0.00(0.00-0.00)$ & 1.000 \\
\hline Clostridium cluster IV & $2.70(1.83-6.78)$ & $4.05(2.92-8.52)$ & 0.529 \\
\hline Clostridium subcluster XIVa & $9.77(7.17-14.83)$ & $12.10(10.66-19.08)$ & 0.813 \\
\hline Clostridium cluster IX & $1.04(0.27-3.32)$ & $1.96(0.51-3.04)$ & 0.578 \\
\hline Clostridium cluster XI & $0.32(0.00-4.12)$ & $0.00(0.00-3.53)$ & 0.201 \\
\hline Clostridium cluster XVIII & $1.75(1.12-3.76)$ & $1.32(0.79-2.87)$ & 0.059 \\
\hline Others & $5.09(3.24-7.54)$ & $6.52(5.10-8.80)$ & 0.469 \\
\hline Shannon's diversity index & $1.53(1.45-1.90)$ & $1.69(1.60-1.90)$ & 0.813 \\
\hline
\end{tabular}

Emboldened P-values indicate statistically significant results. Values are presented as the median (interquartile range). P-values were determined by the Wilcoxon signed-rank test. For organic acids, the percentage of samples above the limit of quantification is shown. Median (IQR) levels of fecal organic acids were determined only for the samples above the limit of quantification. CAI, clinical activity index; UCEIS, ulcerative colitis endoscopic index of severity; GSRS, gastrointestinal symptom rating scale; IQR, interquartile range; T-RFLP, terminalrestriction fragment length polymorphism. 

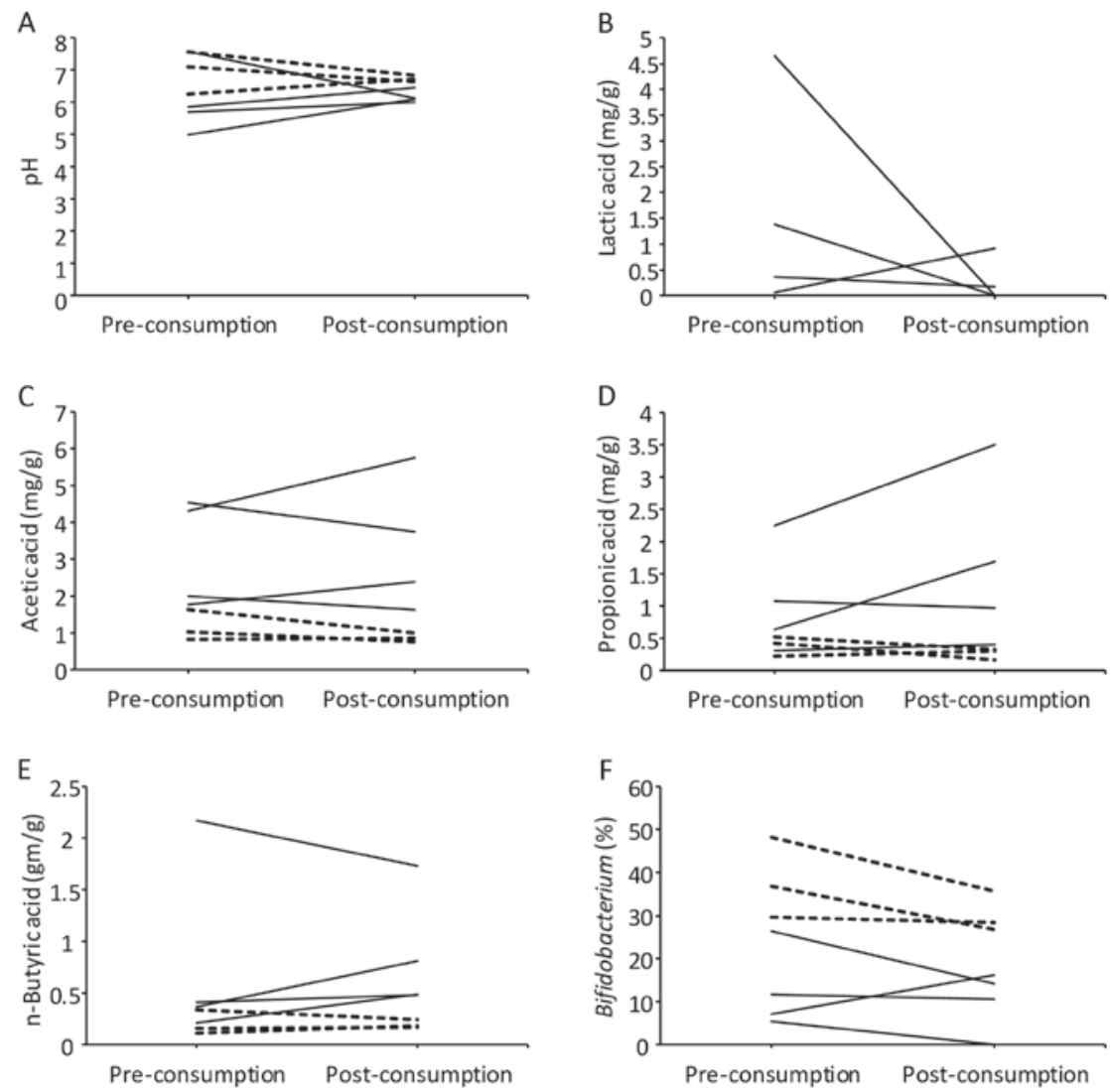

Figure 3. Changes in each parameter pre and post- consumption. Shown are changes in (A) pH, levels of the organic acids (B) lactic acid, (C) acetic acid, (D) propionic acid and (E) n-butyric acid, and (F) Bifidobacterium occupancy. The solid lines indicate patients in which Rachmilewitz CAI declined following consumption (responders); the broken lines indicate patients in which Rachmilewitz CAI remained unchanged or increased (non-responders). The zero on the Y-axis represents values under the limit of quantification. The value of the limit of quantification of each parameter was as stated in the Results section. CAI, clinical activity index.

for personal reasons. Consequently, a total of 7 subjects ( 5 in Group A and 2 in Group B) completed the 8-week consumption regimen (Fig. 1). Due to the dropout rate, the total cohort was treated as a single patient group for the following analyses.

Changes in clinical symptoms. Fig. 2 presents the Rachmilewitz CAI of all subjects enrolled in the study. No significant changes were observed in the clinical activity and endoscopic severity prior to and following consumption (Table III). Regarding the GSRS, there was significant improvement in the total GSRS score $(\mathrm{P}=0.042)$ and for Question 12 'Have you been bothered by loose stools during the past week? [If your stools (motions) have been alternately hard and loose, this question only refers to the extent you have been bothered by the stools being loose.]' ( $\mathrm{P}=0.048$; Table III). No significant changes were observed in the T-RFLP occupancy, $\mathrm{pH}$, fecal organic acids and microbiota prior to and following the consumption period (Table III).

Comparison between responders and non-responders. As depicted in Fig. 2, 4 subjects exhibited improvement of their Rachmilewitz CAI (responders), whereas 3 experienced either unchanged or exacerbated condition (non-responders). Fig. 3 presents the analytic results of fecal $\mathrm{pH}$, organic acids and microbiota. Although no statistical significance was present due to the limited number of subjects, the responders appeared to start at lower $\mathrm{pH}$ than the non-responders, and while they mostly exhibited an increased $\mathrm{pH}$ following consumption, they still tended to exhibit lower $\mathrm{pH}$ following consumption compared with the non-responders (Fig. 3A). Regarding the measurement of organic acids, the value of the limit of quantification was $0.05 \mathrm{mg} / \mathrm{g}$ for succinic acid, lactic acid, acetic acid and propionic acid, and $0.10 \mathrm{mg} / \mathrm{g}$ for formic acid, iso-butyric acid, n-butyric acid, iso-valeric acid and n-valeric acid. Formic acid and n-valeric acid were not detected in all samples. While the levels of lactic acid tended to decrease following consumption of the fermented vegetable beverage among the responders, lactic acid was not detected in the non-responders prior to consumption (Fig. 3B). The levels of acetic acid, propionic acid and n-butyric acid tended to be lower prior to consumption in the non-responders than in the responders (Fig. 3C-E). The levels of these organic acids did not significantly change following consumption in responders and non-responders (Table III). The microbiota analysis revealed that the occupancy of Bifidobacterium tended to be lower in the responders compared with in the non-responders prior to and following consumption (Fig. 3F).

\section{Discussion}

The present study observed that the 8-week consumption of the fermented vegetable beverage by UC patients marginally improved loose stool symptoms but did not affect the disease activity of UC itself. The organic acid analysis revealed that 
the levels of acetic acid, propionic acid and n-butyric acid tended to be continuously higher in the responders than in the non-responders.

Per serving (100 g), the fermented vegetable beverage (Yasai no Senshi ${ }^{\circledR}$ ) contains 8 varieties of vegetable (equivalent to $150 \mathrm{~g}$ in weight) and approximately $5 \times 10^{10}$ colony-forming units (CFU) of Pc. pentosaceus. Pc. pentosaceus was classified as Lactobacillales for the T-RFLP analysis. However, the occupancy of Lactobacillales was not observed to increase following consumption of the beverage. A previous study reported that $10^{6}-10^{7} \mathrm{CFU} / \mathrm{g}$ of $P c$. pentosaceus was detected in the feces of healthy subjects who had consumed Yasai no Senshi ${ }^{\circledR}$ (19). As the Pc. pentosaceus bacterial count was not proportionally high within feces (19), the results of the T-RFLP analysis may not have reflected their presence.

Regarding organic acid analysis, it has been reported that the levels of acetic acid, propionic acid and butyric acid are higher in healthy individuals compared with in UC patients (25-28), whereas the levels of lactic acid are reduced $(26,29)$. In the present study, the responders tended to continuously exhibit higher levels of acetic acid, propionic acid and n-butyric acid; while their levels of lactic acid tended to decrease following consumption of the fermented vegetable beverage. While no significant differences were observed in the index of UC activity between the responders and non-responders, the organic acid analysis revealed that the responders tended to exhibit higher levels of acetic acid, propionic acid and n-butyric acid prior to consumption. This result suggests that the responders probably had an intestinal environment similar to healthy individuals compared with the non-responders even prior to consuming the fermented vegetable beverage.

The gut microbiota analysis revealed that the occupancy of Bifidobacterium tended to be lower in the responders, particularly following consumption. The levels of lactic acid also tended to decrease following consumption. The low occupancy of Bifidobacterium in the responders is consistent with the results of an FMT study targeting UC patients (14). Another previous study indicated that increased levels of lactic acid coincided with exacerbated UC symptoms (29). The source of this lactic acid is considered to be Bifidobacterium (30). Furthermore, another study reported a higher occupancy of Bifidobacterium in UC patients compared with in healthy individuals (3). Given these findings, it is possible that the consumption of the fermented vegetable beverage improved the intestinal environment.

Following consumption of the fermented vegetable beverage, the present subjects experienced improvement in loose stool symptoms. In addition to the effects of Pc. pentosaceus, the symbiotic effects of soluble dietary fiber may have contributed to this improvement.

The present study has limitations due to its single-center nature. Therefore, the number of patients enrolled in this study was limited. Although cases were randomly assigned to two groups, the dropout rate only allowed analysis of a single group; a total of 3 out of 11 patients dropped out, though the effect on the validity of the statistical analysis is unknown. Furthermore, when comparing between the responders and non-responders, statistical significance could not be detected due to the limited size of each group.

In conclusion, the fermented vegetable beverage appeared to ameliorate loose stool symptoms, although the activity of
UC did not improve. Acquisition of further clinical case data is expected to confirm the present findings.

\section{Acknowledgements}

Not applicable.

\section{Funding}

The present study was contracted research between Shiga University of Medical Science (Otsu, Japan) and Otsuka Foods Co., Ltd. (Osaka, Japan). Otsuka Foods supplied the fermented vegetable beverage (Yasai no Senshi ${ }^{\circledR}$ ), and expenses for endoscopic examination and fecal microbiota and organic acid analyses.

\section{Availability of data and materials}

The datasets used and/or analyzed during the current study are available from the corresponding author on reasonable request.

\section{Authors' contributions}

Study concept and design: SB and AA; acquisition of patient data: SB, KT, HI and AN; statistical analysis: SB; drafting of the manuscript: $\mathrm{SB}$ and $\mathrm{KT}$; data interpretation and revision of the manuscript: SB, KT, HI, AN, MK, OI, MSu, MSa and AA.

\section{Ethics approval and consent to participate}

The present study was an open-label, randomized controlled trial with approval of the Ethics Committee of Shiga University of Medical Science, Otsu, Japan (26-205, UMIN000019753). All participants provided written consent to participate in this study.

\section{Consent for publication}

The consent form signed by participants included agreement on the publishing of relevant data following anonymization of personal information.

\section{Competing interests}

The present study was conducted in collaboration with Otsuka Foods Co., Ltd. (Osaka, Japan), the manufacturer and distributer of the fermented vegetable beverage (Yasai no Senshi ${ }^{\circledR}$ ). All authors declare that they have no other competing interests.

\section{References}

1. Chan HC and Ng SC: Emerging biologics in inflammatory bowel disease. J Gastroenterol 52: 141-150, 2017.

2. Andoh A, Sakata S, Koizumi Y, Mitsuyama K, Fujiyama Y and Benno Y: Terminal restriction fragment length polymorphism analysis of the diversity of fecal microbiota in patients with ulcerative colitis. Inflamm Bowel Dis 13: 955-962, 2007.

3. Nishino K, Nishida A, Inoue R, Kawada Y, Ohno M, Sakai S, Inatomi O, Bamba S, Sugimoto M, Kawahara M, et al: Analysis of endoscopic brush samples identified mucosa-associated dysbiosis in inflammatory bowel disease. J Gastroenterol 53: 95-106, 2018.

4. Guandalini S: Update on the role of probiotics in the therapy of pediatric inflammatory bowel disease. Expert Rev Clin Immunol 6: 47-54, 2010. 
5. Abdin AA and Saeid EM: An experimental study on ulcerative colitis as a potential target for probiotic therapy by Lactobacillus acidophilus with or without 'olsalazine'. J Crohn's Colitis 2: 296-303, 2008

6. Ishikawa H, Akedo I, Umesaki Y, Tanaka R, Imaoka A and Otani T: Randomized controlled trial of the effect of bifidobacteriafermented milk on ulcerative colitis. J Am Coll Nutr 22: 56-63, 2003.

7. Kato K, Mizuno S, Umesaki Y, Ishii Y, Sugitani M, Imaoka A, Otsuka M, Hasunuma O, Kurihara R, Iwasaki A and Arakawa Y: Randomized placebo-controlled trial assessing the effect of bifidobacteria-fermented milk on active ulcerative colitis. Aliment Pharmacol Ther 20: 1133-1141, 2004.

8. Tursi A, Brandimarte G, Giorgetti GM, Forti G, Modeo ME and Gigliobianco A: Low-dose balsalazide plus a high-potency probiotic preparation is more effective than balsalazide alone or mesalazine in the treatment of acute mild-to-moderate ulcerative colitis. Med Sci Monit 10: PI126-PI131, 2004.

9. Bibiloni R, Fedorak RN, Tannock GW, Madsen KL, Gionchetti P, Campieri M, De Simone C and Sartor RB: VSL\#3 probiotic-mixture induces remission in patients with active ulcerative colitis. Am J Gastroenterol 100: 1539-1546, 2005.

10. Soo I, Madsen KL, Tejpar Q, Sydora BC, Sherbaniuk R, Cinque B, Di Marzio L, Cifone MG, Desimone C and Fedorak RN: VSL\#3 probiotic upregulates intestinal mucosal alkaline sphingomyelinase and reduces inflammation. Can J Gastroenterol 22: 237-242, 2008

11. Sood A, Midha V, Makharia GK, Ahuja V, Singal D, Goswami P and Tandon RK: The probiotic preparation, VSL\#3 induces remission in patients with mild-to-moderately active ulcerative colitis.Clin Gastroenterol Hepatol 7: 1202-1209, 2009.

12. Tursi A, Brandimarte G, Papa A, Giglio A, Elisei W, Giorgetti GM Forti G, Morini S, Hassan C, Pistoia MA, et al: Treatment of relapsing mild-to-moderate ulcerative colitis with the probiotic VSL\#3 as adjunctive to a standard pharmaceutical treatment: A double-blind, randomized, placebo-controlled study. Am J Gastroenterol 105: 2218-2227, 2010.

13. Shen J, Zuo ZX and Mao AP: Effect of probiotics on inducing remission and maintaining therapy in ulcerative colitis, Crohn's disease, and pouchitis: Meta-analysis of randomized controlled trials. Inflamm Bowel Dis 20: 21-35, 2014.

14. Nishida A, Imaeda H, Ohno M, Inatomi O, Bamba S, Sugimoto M and Andoh A: Efficacy and safety of single fecal microbiota transplantation for Japanese patients with mild to moderately active ulcerative colitis. J Gastroenterol 52: 476-482, 2017.

15. Paramsothy S, Kamm MA, Kaakoush NO, Walsh AJ, van den Bogaerde J, Samuel D, Leong RWL, Connor S, Ng W, Paramsothy $\mathrm{R}$, et al: Multidonor intensive faecal microbiota transplantation for active ulcerative colitis: A randomised placebo-controlled trial. Lancet 389: 1218-1228, 2017.

16. Faith JJ, Guruge JL, Charbonneau M, Subramanian S, Seedorf H, Goodman AL, Clemente JC, Knight R, Heath AC, Leibel RL, et al: The long-term stability of the human gut microbiota. Science 341: 1237439, 2013.
17. Li SS, Zhu A, Benes V, Costea PI, Hercog R, Hildebrand F, Huerta-Cepas J, Nieuwdorp M, Salojärvi J, Voigt AY, et al: Durable coexistence of donor and recipient strains after fecal microbiota transplantation. Science 352: 586-589, 2016.

18. Nishida A, Inoue R, Inatomi O, Bamba S, Naito Y and Andoh A: Gut microbiota in the pathogenesis of inflammatory bowel disease. Clin J Gastroenterol 11: 1-10, 2018.

19. Kumemura M, Saito M, Okamatsu H, Domae T and Isono T: Survival of Pediococcus pentosaceus species in the gastrointestinal tract and the effect of cultured vegetable drink on fecal microflora in human. J Intestinal Microbiol 16: 139-143, 2002.

20. Schroeder KW, Tremaine WJ and Ilstrup DM: Coated oral 5-aminosalicylic acid therapy for mildly to moderately active ulcerative colitis. A randomized study. N Engl J Med 317: 1625-1629, 1987.

21. Rachmilewitz D: Coated mesalazine (5-aminosalicylic acid) versus sulphasalazine in the treatment of active ulcerative colitis: A randomised trial. BMJ 298: 82-86, 1989.

22. Dimenäs E, Glise H, Hallerbäck B, Hernqvist H, Svedlund J and Wiklund I: Quality of life in patients with upper gastrointestinal symptoms. An improved evaluation of treatment regimens? Scand J Gastroenterol 28: 681-687, 1993.

23. Andoh A, Imaeda H, Aomatsu T, Inatomi O, Bamba S, Sasaki M, Saito Y, Tsujikawa T and Fujiyama Y: Comparison of the fecal microbiota profiles between ulcerative colitis and Crohn's disease using terminal restriction fragment length polymorphism analysis. J Gastroenterol 46: 479-486, 2011.

24. Nagashima K, Hisada T, Sato M and Mochizuki J: Application of new primer-enzyme combinations to terminal restriction fragment length polymorphism profiling of bacterial populations in human feces. Appl Environ Microbiol 69: 1251-1262, 2003.

25. Nemoto H, Kataoka K, Ishikawa H, Ikata K, Arimochi H, Iwasaki T, Ohnishi Y, Kuwahara T and Yasutomo K: Reduced diversity and imbalance of fecal microbiota in patients with ulcerative colitis. Dig Dis Sci 57: 2955-2964, 2012.

26. Vernia P, Gnaedinger A, Hauck W and Breuer RI: Organic anions and the diarrhea of inflammatory bowel disease. Dig Dis Sci 33: 1353-1358, 1988.

27. Takaishi H, Matsuki T, Nakazawa A, Takada T, Kado S, Asahara T, Kamada N, Sakuraba A, Yajima T, Higuchi H, et al: Imbalance in intestinal microflora constitution could be involved in the pathogenesis of inflammatory bowel disease. Int J Med Microbiol 298: 463-472, 2008

28. Machiels K, Joossens M, Sabino J, De Preter V, Arijs I, Eeckhaut V, Ballet V, Claes K, Van Immerseel F, Verbeke K, et al: A decrease of the butyrate-producing species Roseburia hominis and Faecalibacterium prausnitzii defines dysbiosis in patients with ulcerative colitis. Gut 63: 1275-1283, 2014.

29. Vernia P, Caprilli R, Latella G, Barbetti F, Magliocca FM and Cittadini M: Fecal lactate and ulcerative colitis. Gastroenterology 95: 1564-1568, 1988

30. Berlec A, Ravnikar M and Strukelj B: Lactic acid bacteria as oral delivery systems for biomolecules. Pharmazie 67: 891-898, 2012. 